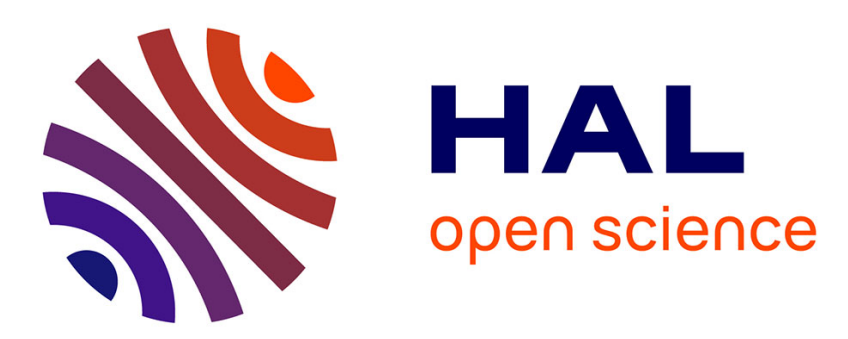

\title{
A probabilistic atlas of the human pyramidal tract in 410 healthy participants
}

Quentin Chenot, Nathalie Tzourio-Mazoyer, Francois Rheault, Maxime

Descoteaux, Fabrice Crivello, Laure Zago, Emmanuel Mellet, Gaël Jobard, Marc Joliot, Bernard Mazoyer, et al.

\section{To cite this version:}

Quentin Chenot, Nathalie Tzourio-Mazoyer, Francois Rheault, Maxime Descoteaux, Fabrice Crivello, et al.. A probabilistic atlas of the human pyramidal tract in 410 healthy participants. 2018. hal01696385

\section{HAL Id: hal-01696385 \\ https://hal.science/hal-01696385}

Preprint submitted on 30 Jan 2018

HAL is a multi-disciplinary open access archive for the deposit and dissemination of scientific research documents, whether they are published or not. The documents may come from teaching and research institutions in France or abroad, or from public or private research centers.
L'archive ouverte pluridisciplinaire HAL, est destinée au dépôt et à la diffusion de documents scientifiques de niveau recherche, publiés ou non, émanant des établissements d'enseignement et de recherche français ou étrangers, des laboratoires publics ou privés. 


\title{
A probabilistic atlas of the human pyramidal tract
}

\section{in 410 healthy participants}

\author{
Quentin CHENOT $^{1}$, Nathalie TZOURIO-MAZOYER ${ }^{1}$, François RHEAULT ${ }^{2}$, Maxime \\ DESCOTEAUX², Fabrice CRIVELLO ${ }^{1}$, Laure ZAGO ${ }^{1}$, Emmanuel MELLET ${ }^{1}$, Gaël JOBARD, \\ Marc JOLIOT ${ }^{1}$, Bernard MAZOYER ${ }^{1}$, Laurent PETIT ${ }^{1}$
}
${ }^{1}$ Groupe d'Imagerie Neurofonctionnelle, Institut des Maladies Neurodégénératives - UMR 5293, CNRS, CEA University of Bordeaux, Bordeaux, France
${ }^{2}$ Sherbrooke Connectivity Imaging Lab, University of Sherbrooke, Canada

\section{Corresponding author:}

Laurent PETIT, Groupe d'Imagerie Neurofonctionnelle

Institut des Maladies Neurodégénératives - CNRS UMR 5293

Université de Bordeaux

Centre Broca Nouvelle-Aquitaine - 3ème étage

146 rue Léo Saignat - CS 61292 - Case 28

33076 Bordeaux Cedex

Email: laurent.petit@u-bordeaux.fr 


\section{Abstract}

With the advances in diffusion MRI and tractography, numerous atlases of the human pyramidal tract (РyT) have been proposed but the inherent limitation of tractography to resolve crisscrossed bundles within the centrum semiovale have so far prevented the complete description of the most lateral PyT projections. Here, we combined a precise manual positioning of individual subcortical regions of interest along the descending pathway of the PyT with a new bundle-specific tractography algorithm. This later is based on anatomical priors to improve streamlines tracking in crossing areas. We then extracted both left and right PyT in a large cohort of 410 healthy participants and built a probabilistic atlas of the whole-fanning PyT with a complete description of its most cortico-lateral projections. Clinical applications are envisaged, the whole-fanning probabilistic PyT atlas being likely a better marker of corticospinal integrity metrics than those currently used within the frame of prediction of post-stroke motor recovery. The present probabilistic PyT, freely available, provides an interesting tool for clinical applications in order to locate specific РyТ damage and its impact to the short and long-term motor recovery after stroke.

\section{Keywords}

White matter anatomy; Pyramidal Tract; Corticospinal tract; Corticobulbar tract; Healthy human; Diffusion imaging; Tractography

\section{Acknowledgments}

We are grateful to Dr. Thomas Tourdias for helpful comments and discussion.

\section{Compliance with Ethical Standards}

1) No specific funding to mention;

2) No conflicts of interest;

3) The current research involved human participants.

4) The study was approved by the local ethics committee (CCPRB Basse-Normandie);

5) All participants gave written consent prior to participation in the study. 


\section{Introduction}

One of the challenges in the human brain connectomic field is to improve our knowledge about major long-range white matter (WM) connections and to link them with behavioral measurements, clinical deficits and recovery (Jbabdi and Behrens, 2012; Jbabdi et al., 2015; Maier-Hein et al., 2017). Among them, the pyramidal tract (PyT) is a long descending pathway which is crucial for the performance of voluntary motricity (Nathan and Smith, 1955; Nyberg-Hansen and Rinvik, 1963; Ebeling and Reulen, 1992). The PyT is generally considered to comprise the corticospinal tract (CST), monitoring movements of the limbs and trunk, and the corticobulbar tract (CBT), directing the movement of the face, head and neck (Nieuwenhuys et al., 2008). Note that the "pyramidal" terminology corresponds to the fact that the PyT runs longitudinally within the pyramids of the medulla oblongata, and does not mean that the PyT fibers originate in the pyramidal cells of the cortex.

After its first detailed description (Dejerine and Dejerine-Klumpke, 1901), the pathway of the PyT in the internal capsule has been enriched in a series of papers and accurately localized in the posterior part of the posterior limb of the internal capsule (Englander et al., 1975; Ross, 1980; Kretschmann, 1988; Ebeling and Reulen, 1992). Early neuroimaging studies with T1 and T2 MRI sequences evidenced at this exact location an hypersignal corresponding to the highly myelinated PyT fibers (Curnes et al., 1988; Mirowitz et al., 1989; Yagishita et al., 1994). Recent studies have used this hypersignal as a marker to delineate the CST in order to study its asymmetry (Hervé et al., 2009; Hervé et al., 2011). Below the internal capsule, CST and CBT fibers enter the pons by passing through the feet of the cerebral peduncle (Dejerine and Dejerine-Klumpke, 1901). CST fibers continue their descent in the anterior part of the brainstem, then go along the medulla oblongata within the pyramid (Nathan \& Smith, 1955) before that $90 \%$ of them decussate when entering the spinal cord to finally join their target motoneurons at different levels (Nieuwenhuys et al., 2008). Note that the CBT fibers project to the cranial nerve motor nuclei in the brainstem and most of them do not descend as far as the medulla oblongata. However, from a functional point of view (both 
tracts control voluntary movements) the term "pyramidal tract" has been long used as including corticobulbar as well as corticospinal fibers (Nyberg-Hansen and Rinvik, 1963). Strictly speaking, PyT encompasses all fibers from the cortex to the lower part of the brainstem (Ebeling and Reulen, 1992).

With the emergence of diffusion-weighted magnetic resonance imaging (DWI) and tractography, several atlases of the major white matter pathways have been proposed including rather the CST than the PyT ((Zhang et al., 2010; Thiebaut de Schotten et al., 2011; Yendiki et al., 2011; Rojkova et al., 2016); but see (Archer et al., 2017)). The limitation of DWI-tractography to resolve intricate fiber crossing particularly concerns the PyT for which the most lateral fibers cross within the centrum semiovale, the corpus callosum (CC) and the long-range association pathways, mainly the superior longitudinal fasciculus (SLF) and the arcuate fasciculus (AF). As a matter of fact, the previous atlases did not reveal a comprehensive fanning in the lateral projections of the CST/CBT along the primary motor cortex (Thiebaut de Schotten et al., 2011; Rojkova et al., 2016; Archer et al., 2017).

Bürgel et al. (2006) created the first stereotaxic CST atlas by using staining method in 10 adult post-mortem brains. Although they limited their selection to CST fibers originating from the precentral gyrus, they described a comprehensive fanning at the cortical level and the passage of the CST in the posterior part of the posterior limb of the internal capsule (Bürgel et al., 2006). By using diffusion-weighted tractography, Thiebaut de Schotten et al. (2011) proposed a CST atlas in 40 healthy participants. They evidenced dorso-medial CST streamlines in the precentral gyrus but no lateral cortical fanning. This lack was explained by the limitation of the diffusion tensor model in resolving crossing fibers bottleneck between the SLF, CC and PyT streamlines in the centrum semiovale. Rojkova et al. (2015) used a more advanced model of tractography (spherical deconvolution), and tracked cortical projections along the central sulcus at the individual level. However, they observed that the lateral projections were too seldom to be represented in the probabilistic atlas. More recently, Archer et al. (2017) constructed a template of the sensorimotor area tracts (SMAT) by using probabilistic tractography. Compared to previous tractography studies, these authors 
changed the methodology and seeded from multiple sensory-motor areas instead of the precentral area only. They found direct cortico-spinal streamlines from these frontal areas and obtained a better fanning of cortical projections. However, ventro-lateral projections were still lacking in the probabilistic atlas they constituted with 100 healthy participants of the Human Connectome Project (Archer et al., 2017).

The inherent limitation of diffusion-weighted tractography to resolve crisscrossed bundles within the centrum semiovale has so far prevented the complete description of the most lateral РyT cortical projections. The aim of the present study is to tackle these limitations to establish a probabilistic atlas of the whole-fanning PyT by applying a new bundle-specific tractography algorithm based on anatomical priors (Rheault et al., 2017) to improve streamlines tracking in crossing areas. We also defined dedicated anatomical regions of interest (ROIs) at the individual level in order to obtain a specific and sensitive extraction of the PyT in 410 participants of the BIL\&GIN database (Mazoyer et al., 2016). We therefore built a probabilistic PyT atlas with a complete dorso-lateral fanning in each hemisphere. Because damages of the PyT by stroke, tumor, multiple sclerosis, or cerebral palsy can impact the human motor system, one of the stakes of clinical research is to better evaluate initial PyT damages to better predict short and long-term motor recovery (Groisser et al., 2014; Bigourdan et al., 2016). The present PyT atlas may then be a suitable tool in clinical research to investigate these questions. 


\section{Material and methods}

\section{Participants}

The present study used diffusion-weighted and T1-weighted images of 410 healthy participants from the BIL\&GIN database, balanced in both sex (53\% women) and handedness (49\% left-handers) with a mean age of $29.9( \pm 7.9)$ years and $15.1( \pm 2.6)$ years of education (Mazoyer et al., 2016). All participants gave written consent prior to participation in the study, which was approved by the local ethics committee (CCPRB Basse-Normandie).

\section{MRI acquisitions and processing}

\section{Structural MRI}

High-resolution 3D T1 weighted images were obtained using a 3D-FFE-TFE sequence (TR, $20 \mathrm{~ms}$; TE, $4.6 \mathrm{~ms}$; flip angle, 10; inversion time, $800 \mathrm{~ms}$; turbo field echo factor, 65; Sense factor, 2; field of view, $256 \times 256 \times 180 \mathrm{~mm}$; isotropic voxel, $1 \times 1 \times 1 \mathrm{~mm}^{3}$ ). For each participant, the line between anterior (AC) and posterior (PC) commissures was identified on a mid-sagittal section, and the T1-MRI volume was acquired after orienting the brain in the bi-commissural coordinate system. Each participant T1-MRI volume was warped to its native diffusion space using ANTS linear and nonlinear registration (Avants et al., 2011).

\section{Diffusion MRI}

Diffusion-weighted imaging (DWI) data were acquired using a single-shot spin-echo echoplanar sequence composed of one $b_{0}$ map $\left(b=0 \mathrm{~s} / \mathrm{mm}^{2}\right)$ followed by 21 non-collinear diffusion gradient directions maps $\left(b=1000 \mathrm{~s} / \mathrm{mm}^{2}\right)$, this series was acquired twice by reversing the gradients' polarity. A second series of $44 \mathrm{DWI}$ volumes was acquired to reduce signal to noise ratio. Seventy axial slices parallel to the AC-PC plane were acquired from the bottom of the cerebellum to the vertex with the following imaging parameters: TR $=8500 \mathrm{~ms}$; $\mathrm{TE}=81 \mathrm{~ms}$; angle $=90^{\circ} ;$ SENSE reduction factor $=2.5$; field of view $224 \mathrm{~mm}$; acquisition 
matrix $112 \times 112,2 \times 2 \times 2 \mathrm{~mm}^{3}$ isotropic voxel. 70 axial slices parallel to the AC-PC plane were acquired from the bottom of the cerebellum to the vertex.

Individual raw DWI data were first corrected for eddy current distortion using the FMRIB software library (Smith et al., 2004) and then processed using Dipy-related tools (Garyfallidis et al., 2014) to obtain the diffusion tensor, the fractional anisotropy (FA), 3-direction vector colored FA (RGB) and fibers orientation distribution of spherical harmonics order 6 (fODF, (Descoteaux et al., 2009)) maps. Note that these different maps were upsampled to $1 \mathrm{~mm}^{3}$ spatial resolution using trilinear interpolation (Girard et al., 2014). $B_{0}$, FA and RGB maps were used for the positioning of the regions of interest which delineate the PyT.

\section{Definition and positioning of ROls (Figure 1)}

In order to extract the PyT of each participant $(n=410)$, we manually defined and positioned, in each hemisphere, 3 ROls along its pathway at the level of the internal capsule, the midbrain and the medulla oblongata. The manual delineation of these ROls, their visualization and the filtering of the PyT were performed with TrackVis (http://www.trackvis.org/). Additional anatomical ROls (referred as JHU-...) were used from the JHU template (Zhang et al., 2010) once warped to the native diffusion space of each subject using ANTS linear and non-linear registration.

Internal Capsule ROI (IC-ROI)

The IC-ROI was a disk of $8 \mathrm{~mm}$ of diameter (57 voxels) delineated on a single axial slice. To position this $\mathrm{ROI}$ in the z-axis, the barycenter of the $\mathrm{JHU}$-thalamus was first calculated and its z-coordinates used because it nearly corresponds to the middle of the adjacent internal capsule in the z-axis. The IC-ROI was then centered for each participant on the hypersignal localized on an enhanced thresholded- $b_{0}$ map (Figure 1). To do so, we first binarized the $b_{0}$ map thresholded between 30 and $75 \%$ of its highest value by step of $5 \%$, highlighting the hypersignal at the level of the internal capsule (Figure 1A.1). We also extracted the blue map 
from the RGB color map, i.e. the voxels with a superior-to-inferior main diffusion orientation, thresholded it at $50 \%$ of its highest value and binarized it (Figure 1A.2). A series of enhanced thresholded- $b_{0}$ maps were obtained by overlapping these two maps (Figure 1A.3), highlighting the hypersignal as a single cluster at the level of the internal capsule for a given percentage (for example $55 \%$ in the example illustrated in Figure $1 \mathrm{~A}$ ) on which the IC-ROI was centered Figure 1B.1). The final positioning of the IC-ROI was visually controlled by superimposition on the T1 map (Figure 1B.1).

\section{Midbrain ROI (MB-ROI)}

MB-ROI was a disk of $13 \mathrm{~mm}$ of diameter (129 voxels) delineated on a single axial slice. It was used to discriminate the PyT (descending motor pathway) localized in the anterior part of the midbrain from the lemniscus tract (ascending sensory pathway) localized in the posterior part of the midbrain. To position this ROI in the z-axis, the RGB color map was used to highlight both tracts in blue color (Figure 1B.2). The axial section in which the two tracts were the most distant from each other was then selected, and the ROI was centered on the blue signal located in the anterior part of the midbrain.

\section{Pyramids of the Medulla Oblongata (MO-ROI)}

MO-ROI was a disk of $11 \mathrm{~mm}$ of diameter (97 voxels) delineated on a single axial slice, which was used to select fibers entering in the medulla while not passing through the cerebellum. Note that at this level, the fibers are forming a pyramidal section. To position the MO-ROI in the z-axis, the T1 and RGB color map were used at the level of the medulla oblongata. The criterion of selection was that the white matter of the medulla oblongata had to be separated from the white matter of the cerebellum and had to form a pyramidal section in the axial T1 map (Figure 1B.3). For each participant, we then positioned the ROI to encompass the pyramids, which appeared in blue on the RGB map at the level of the chosen axial T1 slice (Figure 1B.3). 


\section{Anatomical location of ROIS in the MNI-space}

The three ROls along the PyT pathway for both hemispheres were warped in the MNI-space by using ANTS linear and non-linear registration. The center of mass of each ROI was calculated for the three axes $(x, y, z)$, for each hemisphere and for each of the 410 participants.

Intra-operator reliability for ROl's positioning

To ensure that the ROls positioning was robust, we performed a reproducibility analysis in a random sub-sample of 40 out of the 410 participants. ROls were positioned twice by the same operator after shuffling the participants' images and their side, using the same methodology. ROls coordinates were calculated in order to perform an intraclass correlation between the first and the second positioning and to evaluate the reproducibility. The Euclidean distance was also calculated for both positioning.

\section{Building a PyT template to be used as bundle of interest (Figure 2)}

We computed the whole brain tractogram of 39 participants. Following a procedure that has been previously fully described (Hau et al., 2017), each tractogram was built using constrained-spherical deconvolution and particle-filter tractography with anatomical priors (Girard et al., 2014). In brief, each whole tractogram was composed of about 1.5 millions of streamlines seeded from the white/gray-matter interface (10 seeds/voxel, Figure 2A.1) and terminating within the gray matter with a streamline's length between 10 and $250 \mathrm{~mm}$. We then extracted the PyT in each hemisphere of these 39 participants by using their manuallydefined IC-, MB- and MO-ROls as points of passages and a JHU-fronto-parietal ROI as termination for the streamlines (Figure 2A.2). This later consisted in a single fronto-parietal $\mathrm{ROI}$ in each hemisphere defined by merging all frontal and parietal regions of the $\mathrm{JHU}$ template (including superficial white matter regions which were merged with their corresponding gyral region). An outlier removal algorithm was applied on each PyT to prune 
anatomical outlier streamlines (Côté et al., 2015), i.e streamlines with multiple curves and loops. Finally, we set a termination-enhanced strategy by keeping the streamlines only ending in the gray matter part of the JHU-fronto-parietal ROI (Figure 2A.3).

Second, we build a N39-PyT template from these initial PyTs. We first performed an ANTs template construction (Avants et al., 2011) using the FA maps of the 39 participants to obtain a N39-FA template used as a common space for the N39-PyT template (Figure 2B.1). The nonlinear deformation was then applied to the streamlines of each of the 39 PyTs (Figure 2B.2). Since only the shape and position of the streamlines are relevant for the construction of the РуT template, redundant streamlines were removed using QuickBundles, a hierarchical clustering algorithm and the minimal direct-flip distance operator (Garyfallidis et al., 2012). Each PyT was decimated by discarding non-essential streamlines, i.e. those that can be removed with no loss of information about the shape or position of the PyT streamlines (Rheault et al., 2017). This operation helps to reduce in the subsequent optimized tractography the biases caused by an over-representation of straight streamlines in the most medial part of PyT and an under-representation of fanning in the most lateral part of PyT.

\section{Enhancing orientation distribution within the bundle of interest (Figure 2C).}

The N39-PyT template was used as a bundle of interest (BOI) to optimize the final tractography of the PyT in the 410 participants. Since the N39-PyT template was created in a common space, a nonlinear deformation was needed to apply these priors to each participant. We computed the nonlinear deformation matrix between the N39-FA template and the FA map for each of the 410 participants and applied it to the N39-PyT template to displace the N39-PyT streamlines in each individual diffusion space. The next optimization consisted in a voxel-wise weighting of fODF lobes according to the direction of the streamlines from the N39-PyT template (Rheault et al., 2017). The method is based on a track orientation distribution (TOD) map (Dhollander et al., 2014). It produces a new set 
fODF where the influence of lobes with the same general direction as the PyT is slightly increased, while the influence of lobes with a distinct direction is slightly decreased. This result in a more efficient tracking, with less abrupt changes in direction and a better performance in crossing area resulting in an improved coverage (see Discussion).

Weighted-fODF tractography of the PyT within the internal capsule ROI (Figure 2D).

At that stage, the streamlines of the N39-PyT template were used as mask to constrain the tracking in the global shape of the PyT (yellow part of Figure 2C-2). A final tractography was then performed by using the particle-filter tractography algorithm within this new tracking mask and the weighted fODF map previously mentioned. The seeding was initiated in the right and left IC-ROI (57 voxels both) with 1000 seeds per voxel. The length of the streamlines was set at a minimum of 80 and a maximum of $180 \mathrm{~mm}$. If not otherwise specified, we used the default parameters. For each of the 410 participants, this created a PyT raw tractogram (Figure 2D-2) on which we applied the 3 manually positioned ROls to select streamlines (Figure 2D-3). The number of streamlines and the volume in the native space (after binarization, Figure 2D-4) were calculated for each participant.

\section{PyT atlases}

Each of the 410 left and right PyT was normalized to the MNI space using the ANTS inverse warp and affine matrices that were used to register the JHU template to the diffusion space in order to concatenate them in a N410-PyT tractogram composed of about 4.4 and 5 millions of streamlines for left and right hemisphere, respectively. A lot of these streamlines once normalized were spatially identical. We thus reduced the number of streamlines composing each N410-PyT by removing redundant streamlines across participants using a hierarchical clustering algorithm and the minimal direct-flip (MDF) distance operator (Garyfallidis et al., 2012). 
Each individual PyT tractogram was also binarized, then normalized to the MNI space, to build a probabilistic atlas of the PyT following the method previously applied to create histology and tractography atlases WM tracts (Bürgel et al., 2006; Thiebaut de Schotten et al., 2011). To do so, the 410 binarized PyT maps were then summed and set to a probabilistic map between 0 and 100\% overlap.

\section{Statistical analysis: PyT asymmetry}

Statistical analyses were performed using JMP 11 (www.jmp.com, SAS Institute Inc., 2012). To test the РуT anatomical asymmetry, a repeated MANOVA was computed on 405 participants (note that 5 participants were removed from the analysis because they were inverted left handed). The repeated variable was the hemispheric PyT volume (Left vs Right) in the individual space. Adjustment variables were the Manual Preference (Left vs. Right), Gender (Men vs. Women), Age and the Cerebral Volume. 


\section{Results}

\section{Mean anatomical localization of the 3 ROIs in the MNI space (Table 1)}

Mean coordinates of the center of the IC-ROI were $x=-22.3$ (standard deviation $=1.1$ ), $y=-$ 16.0 (1.3), $z=4.4(1.1)$ on the left, and $x=22.8(1.1), y=-16.0(1.2), z=5.0$ (1.1) on the right. These coordinates in the MNI space actually correspond to the posterior part of the posterior limb of the internal capsule for both hemispheres.

For the MB-ROI, mean coordinates were $x=-6.7(1.2), y=-22.5(1.8), z=-33.9(2.02)$ on the left and $x=7.0(1.1), y=-22.6(1.8), z=-33.6(2.0)$ on the right. In the MNI space, these coordinates were actually in the anterior part of the Midbrain.

For the MO-ROI, mean left coordinates were $x=-4.6(1.1), y=-27.5(1.9), z=-43.0(1.9)$ and mean right: $x=4.9(1.1), y=-27.5(1.8), z=-43.2(1.9)$. In the MNI space, these coordinates correspond to the anterior part of the medulla oblongata.

\section{Inter-operator ROIs positioning reliability (Table 2)}

The reproducibility of ROl's positioning was consistent between the first and second positioning in terms of coordinates. Intraclass correlation coefficients computed were very satisfying being superior to .90 for all ROls and for all incidences $(x, y, z)$. Mean Euclidean distances between the ROls were between 0.61 and $1.15 \mathrm{~mm}$, which correspond approximately to a difference of one voxel, which have a $1 \times 1 \times 1 \mathrm{~mm}^{3}$ resolution.

\section{Description of the PyT atlas}

Both left and right PyTs were obtained in the 410 participants. Figures 3 shows overall concatenated left and right N410-PyTs. Thanks to the manual positioning of the different ROIs along the PyT pathways in each participant, each of the left and right 410 PyT descended through the centrum semiovale, passed through the posterior part of IC, crossed 
the anterior part of the cerebral peduncle and reached the MO through the anterior part of MB. At the cortical level, we observed a whole-fanning of the PyT around all the convexity of the peri-central cortex. Figure 4 shows the overall distribution of the PyT streamlines in the different frontal and parietal gyri that were initially included in the fronto-parietal JHU ROI (see Methods). The largest proportion of cortical termination was found overwhelmingly in the precentral gyrus ( $>50 \%$, Figure $4 \mathrm{~B}$ ), then in the postcentral gyrus $(>26 \%)$, the superior frontal gyrus ( $>13 \%$ ) and to a much lesser extent the superior parietal, supramarginal, middle and inferior frontal gyri. Coronal, axial and sagittal views of these PyT projection per gyri are available in supplementary materials.

It is noteworthy that after a well-ordered cortical organization the streamlines seem to intermingle at the level of the internal capsule while making a twist, the "blueish" streamlines passing in front of the "greenish" streamlines (black arrows in Figure 4A).

\section{PyT volume}

The analysis of individual hemispheric volumes showed a mean volume of $35775 \pm 4002$ $\mathrm{mm}^{3}$ for the left PyT, and $38055 \pm 4380 \mathrm{~mm}^{3}$ for the right PyT. The MANOVA revealed that this asymmetry is significant $(F=181.0, p<0.0001)$. There was no effect of Manual Preference on PyT asymmetry $(F=2.7, p=0.10)$, left and right-handers showing the same volume for each hemisphere (Right handed, left hemisphere $=35620 \pm 3865$; right hemisphere $=37661 \pm 4104$; Left handed, left hemisphere $=35932 \pm 4140$; right hemisphere $=38450 \pm 4620)$. As expected, there was an effect of the cerebral volume $(F=75.1, p<$ 0.0001) with the РyT volume increasing with the cerebral volume. There was an effect of the gender, with men having a bigger PyT volume than women $(F=4.6, p=0.03$; women, left hemisphere $=34333 \pm 3803$; right hemisphere $=36477 \pm 4170$; men, left hemisphere $=$ $37274 \pm 3645$; right hemisphere $=39694 \pm 3982)$ and no age effect $(F=0.1, p=0.78)$. 


\section{Description of the PyT probabilistic atlas}

The probabilistic PyT atlas had a fanning of cortical projections in the superior, medium and inferior parts of the pre- and post-central gyrus (Figure 5) visible on low (10-30\%) as well as high thresholds (70-80\%), especially in $-12,-16$ and -20 coordinates on coronal slices, and in +24 to +60 on axial slices. The superior cortical projections were localized on the posterior coronal planes $(-20,-24,-28,-32)$ whereas the inferior cortical projections were localized more anteriorly $(-4,-8,-12,-16)$. This antero-posterior shift in the dorso-medial direction corresponds to the orientation of the Rolandic sulcus and both precentral and postcentral gyri where the PyT cortical projections were by far found. Figure 6 illustrates the extent of the cortical projection at the different thresholds (from 10\% to $90 \%$ ) and Table 3 lists the volume of the PyT atlas at these different thresholds for both hemispheres. We observed that the right probabilistic PyT atlas was larger than the left one in the same range whatever the threshold (about 2.7\%). Note also that the volumes obtained at the threshold of $40 \%$ (left: $35045 \mathrm{~mm}^{3}$; right: $37052 \mathrm{~mm}^{3}$ ) were similar to the mean volume observed and the individual level (left: $35775 \mathrm{~mm}^{3}$; right: $38055 \mathrm{~mm}^{3}$ ).

As expected, the probabilistic PyT atlas descends through the corona radiata to reach the posterior part of the posterior limb of the internal capsule, between the thalamus and the basal ganglia (axial coordinates 0 and +12 , Figure 5 ). It crosses the cerebral peduncle in its anterior part, the highest thresholds (> $80 \%$ ) showing that it occupies the central part of the feet of the cerebral peduncle (axial coordinates -12, Figure 5). Lowermost, the PyT atlas is centered in the anterior part of the midbrain until it reaches the medulla oblongata (Figure 6). 


\section{Discussion}

We first tracked both left and right PyT in a large cohort of 410 healthy participants by combining a precise manual positioning of specific $\mathrm{ROI}$ along its descending pathway with a new approach of bundle-specific tractography. Then we built a probabilistic PyT atlas from this large cohort with a complete description of its most cortico-lateral projections while previous comparable atlases were restricted to its most medio-dorsal part (Catani and Thiebaut de Schotten, 2008; Zhang et al., 2010; Thiebaut de Schotten et al., 2011; Rojkova et al., 2016; Archer et al., 2017).

\section{PyT cortical projections}

We observed a complete fanning following the central sulcus curvature from the mediodorsal to the ventro-lateral part, closely similar to the one previously described in the dissection and in the histology literature (Dejerine and Dejerine-Klumpke, 1901; Ebeling and Reulen, 1992; Bürgel et al., 2006; Nieuwenhuys et al., 2008) and covering the entire sensorimotor homunculus originally described by Penfield (Penfield and Boldrey, 1937). The presence of a large amount of ventro-lateral projections was striking considering the difficulty of tracking in the centrum semiovale reported in the literature (Farquharson et al., 2013), where multiple bundles are crossing. This observation is likely to result from the use of the CSD-PFT algorithm (Girard et al., 2014), but especially with the weighting of the fODF based on a PyT template, which allowed a better tracking of PyT streamlines in the centrum semiovale (Rheault et al., 2017). These ventro-lateral projections represent a significant improvement compared to previous CST/PyT extracted using individual tractography (Kumar et al., 2009; Zolal et al., 2012; Groisser et al., 2014; Angstmann et al., 2016; Bigourdan et al., 2016) and also compared to the different previous probabilistic CST/PyT atlases (Zhang et al., 2010; Thiebaut de Schotten et al., 2011; Rojkova et al., 2016; Archer et al., 2017). 
We also observed that the PyT cortical projections, namely the corticospinal and corticobulbar tracts, originated in areas beyond the primary motor cortex (M1) along the Rolando sulcus, and even beyond the precentral gyrus. It is consistent with previous studies in non-human primates which showed that the PyT originates from M1 but also from premotor and parietal areas (Armand, 1982; Dum and Strick, 1991; Galea and Darian-Smith, 1994). In humans, the exact extent of PyT cortical origins is still under question (Archer et al., 2017). A post-mortem study has estimated that the majority of PyT fibers (40-60\%) originates from the precentral gyrus in human (Jane et al., 1967; Ebeling and Reulen, 1992), a proportion that we also observed since the most represented PyT projection also originated by far from the precentral gyrus in each hemisphere (Figure 4B). The remainder projections first concerned both postcentral and superior frontal gyri then, to a lesser extent, the posterior parts of the middle and inferior frontal gyri, the supramarginal and superior parietal gyri. In their recent study, Archer et al. (2017) addressed this issue in a different manner by tracking descending sensorimotor tracts from motor and premotor ROls defined by functional brain imaging (Mayka et al., 2006), hinting that both CST and CBT cortical origins certainly extended beyond M1. Similar results were originally described using functionally-defined motor and premotor seedings (Seo and Jang, 2013). The extent of cortical PyT origins is an important question since the existing CST/PyT atlases choose to restrict the cortical origins to the precentral gyrus (Bürgel et al., 2006; Zhang et al., 2010; Thiebaut de Schotten et al., 2011; Rojkova et al., 2016), which does not seem to correspond to the exact anatomical definition of the PyT. Further investigations including blunt Klingler microdissection ideally combined with diffusion-weighted tractography are needed to investigate this issue.

\section{PyT anatomical course}

As expected since the seeding IC-ROI was positioned at that location in each of the 410 participants, the present PyT atlas crossed the posterior part of the posterior limb of the internal capsule as evidenced in previous dissection studies (Englander et al., 1975; Ross, 
1980; Kretschmann, 1988). It is noteworthy that its mean location both in terms of the mean coordinates of the IC-ROI and the probabilistic PyT map (Figure 5) was exactly the same that in the microscopically defined stereotaxic atlas of the CST (restricted to the M1 projection) (see Figure 7 in (Bürgel et al., 2006)).

We observed that after a well-ordered cortical organization the streamlines were intermingled at the level of the internal capsule while making a twist, the "blueish" medial streamlines with a more medial origin passing in front of the "greenish" streamlines (black arrows in Figure 4A). This is very consistent with previous anatomical description of the PyT course (Nathan and Smith, 1955). These authors wrote "From the precentral gyrus, the fibers come together to form a band on the internal capsule. To narrow down to pass through a band-like gap, the fibers undergo a screw-like gyration, the fibers from the inferior part of the gyrus coming to lie most medially and those from the paracentral lobule coming to lie more. (pg. 264)"

The descending course of the PyT atlas into the anterior part of midbrain was also similar to the pathway reported in dissection and histology studies (Dejerine and Dejerine-Klumpke, 1901; Nathan and Smith, 1955; Bürgel et al., 2006). Note that thanks to the accurate ROI positioning in the midbrain, the PyT atlas correctly crossed the feet of the cerebral peduncle which constituted a validation of the PyT pathway, since no ROI was defined at this level to restrict the fibers' pathway.

Our PyT atlas showed a higher resolution in its sub-cortical pathway than previous probabilistic CST/PyT atlases (Thiebaut de Schotten et al., 2011; Rojkova et al., 2016; Archer et al., 2017). This higher accuracy was due to the manual positioning of 3 sub-cortical ROls in each of the 410 participants (posterior part of the posterior limb of the internal capsule; anterior part of the midbrain and pyramids in the medulla oblongata), whereas previous studies applied automated positioning tools and a lower number of sub-cortical ROls. 


\section{PyT asymmetry}

To our knowledge, this is the first report of a rightward РyT volume asymmetry. Previous tractography studies that have quantified the CST/PyT volume in healthy individuals found either an absence of asymmetry or a leftward asymmetry. Kwon et al. (2011) extracted the CST involved in upper limb motricity (hands and fingers) and inferior limb motricity (legs and feet) in 43 right-handed healthy adults using a probabilistic tractography algorithm. Their results showed an absence of asymmetry (Kwon et al., 2011). Kumar et al. (2009) computed a complete tractogram using a deterministic algorithm in 42 children (3-17 years, 33 righthanded). They traced a first ROI in the midbrain and a second in the corona radiata and reported a significant leftward asymmetry of the volume of the CST, with no effects of sex or manual preference (Kumar et al., 2009). However, the use of a deterministic algorithm limited the cortical projections of their CST in the area corresponding to lower limbs motricity. By using a probabilistic tractography algorithm, Seo \& Jang (2011) found no asymmetry in 36 healthy right-handed adults, except for a sub-component originating from the supplementary motor area that was leftward asymmetrical (Seo and Jang, 2013). The discrepancy between these previous studies may be due to methodological limitations such as differences in terms of ROls choice and reconstructed CST-PyT with few or no ventro-lateral cortical projections that would impact on the estimation of the tract volume. The rightward asymmetry we presently report has the advantage of coming from a much larger sample of participants. Finally, the absence of a manual preference effect on the PyT asymmetry allows using the present PyT atlas in any participant whatever his/her handedness.

\section{Limitations}

Although the manual ROls placement allows a fine-grained and specific extraction of the PyT, one may notice that we used ROls with the same size in all participants. While this was the only way to complete a rigorous seeding in the internal capsule, it could be considered as a limitation considering individual variability in brain size. We also have to consider the 
inherent limits in spatial resolution of tractography: the present tractograms were at a millimeter scale $\left(1 \times 1 \times 1 \mathrm{~mm}^{3}\right)$ while axons are at a micro-millimeter scale, meaning that one voxel can contain millions of fibers. As a result, quantification in tractography (whether it is the number of streamlines or the volume of a bundle) has to be supported by anatomical data such as post-mortem dissection or histology studies to be validated (Jones et al., 2013; Maier-Hein et al., 2017). The fact that the descending subcortical pathway of the present PyT atlas is highly similar to histological atlas from Bürgel et al. (2006) can be considered as a first validation step. However, Bürgel's study was restricted to the precentral origin of the CST/PyT that probably underestimated the complete cortical extent of the human PyT.

\section{Conclusion}

In the present study, we built a probabilistic atlas of the PyT in 410 healthy participants by combining (1) manual positioning of sub-cortical regions of interest and (2) an advanced tractography methodology that allowed to track the whole-fanning PyT. We then elaborated the first probabilistic atlas of pyramidal tract having both sub-cortical accurate anatomical pathway and comprehensive fanning projections along the central sulcus. Further works combining diffusion with functional imaging will permit to identify the sub-components of the PyT related to upper limbs motricity, lower limbs motricity or mouth, tongue and face motricity as typically defined in the homunculus (Penfield and Boldrey, 1937). The present probabilistic PyT provide an interesting tool for clinical applications in order to locate specific PyT damage and its impact to the short and long-term motor recovery after stroke (Groisser et al., 2014; Bigourdan et al., 2016; Archer et al., 2017). 
Table 1. Mean (sd) coordinates of the three ROls along the pathway of the CST in the MNI space $(n=410)$.

\section{MNI coordinates}

\begin{tabular}{lccc}
\multicolumn{1}{c}{ ROI } & $\mathbf{x}$ & $\mathbf{y}$ & $\mathbf{z}$ \\
\cline { 2 - 4 } Left Internal Capsule & $-22.32 \pm 1.09$ & $-15.97 \pm 1.28$ & $4.34 \pm 1.13$ \\
Right Internal Capsule & $22.81 \pm 1.11$ & $-15.96 \pm 1.19$ & $4.96 \pm 1.11$ \\
Left Midbrain & $-6.68 \pm 1.19$ & $-22.47 \pm 1.85$ & $-33.86 \pm 2.02$ \\
Right Midbrain & $7.01 \pm 1.13$ & $-22.60 \pm 1.83$ & $-33.62 \pm 2.03$ \\
Left Medulla oblongata & $-4.59 \pm 1.07$ & $-27.50 \pm 1.87$ & $-43.04 \pm 1.89$ \\
& & & \\
Right Medulla oblongata & $4.89 \pm 1.12$ & $-27.48 \pm 1.79$ & $-43.21 \pm 1.89$ \\
\hline
\end{tabular}

Table 2. Intraclass correlation coefficients and mean Euclidean distance (standard deviation) between first and second positioning (individual space).

\begin{tabular}{lcccc}
\hline \multicolumn{1}{c}{ ROI } & X & Y & Z & Euclidean Distance $\left(\mathrm{in} \mathrm{mm}^{3}\right)$ \\
\hline Left internal capsule & .96 & .99 & .99 & $0.61 \pm 0.70$ \\
Right internal capsule & .95 & .99 & .99 & $1.02 \pm 0.70$ \\
Left midbrain & .94 & .99 & .99 & $1.15 \pm 0.73$ \\
Right midbrain & .92 & .99 & .99 & $1.15 \pm 0.77$ \\
Left medulla oblongata & .97 & .98 & .99 & $0.99 \pm 0.68$ \\
Right medulla oblongata & .95 & .99 & .99 & $1.05 \pm 0.61$ \\
\hline
\end{tabular}


Table 3. Volume of the PyT probabilistic atlas thresholded from 10 to $90 \%$ of the 410 participants. For example, $50 \%$ means that 205 out of 410 individual PyTs are included in the volume.

\begin{tabular}{lccc}
\hline Threshold & $\begin{array}{c}\text { Left PyT atlas } \\
\left.\text { (in } \mathrm{mm}^{3}\right)\end{array}$ & $\begin{array}{c}\text { Right PyT atlas } \\
\left.\text { (in } \mathrm{mm}^{3}\right)\end{array}$ & $\begin{array}{c}\text { (R-L)/(R+L) } \\
\text { (in \%) }\end{array}$ \\
\hline $10 \%$ & 70706 & 74271 & 2.5 \\
$20 \%$ & 52366 & 55360 & 2.8 \\
$30 \%$ & 42105 & 44690 & 2.9 \\
$40 \%$ & 35045 & 37052 & 2.8 \\
$50 \%$ & 29326 & 30984 & 2.7 \\
$60 \%$ & 24418 & 25775 & 2.7 \\
$70 \%$ & 19912 & 20971 & 2.6 \\
$80 \%$ & 15552 & 16421 & 2.7 \\
$90 \%$ & 10899 & 11515 & 2.7 \\
\hline
\end{tabular}




\section{Figure legends}

\section{Figure 1}

IC-ROI, MB-ROI and MO-ROI definitions: The binarized $b_{0}$-map thresholded from 30 to $75 \%$ of maximum value (A1) was multiplied by the binarized RGB-based blue map thresholded at $50 \%$ (A2) to build enhanced thresholded- $\mathrm{b}_{0}$ maps to highlight the PyT hypersignal at the level of IC (55\% in A3-B1). MB was positioned on the blue signal in the anterior part of the midbrain (B2) as opposed to the posterior blue signal corresponding to the lemniscus tract. MO was positioned to contain the pyramids, which is apparent in blue on the RGB map at the level of the chosen axial T1 slice (B3).

\section{Figure 2}

A. Thirty-nine whole-tractograms computed by seeding in the GM/WM interface with a CSDPFT model (1-2) on which we applied manually defined ROIs (IC-ROI, MB-ROI, MO-ROI) and cleaned the outliers (3). B. N39-PyT template obtained by concatenating the 39 cleaned PyTs in a common space. C. The N39-PyT template (in yellow) used to weight the initial fODF (1) into optimized fODF for PyT tracking within the N39-PyT mask (in red). D. N39-PyT template-specific tractography with the weighted-fODF within the IC-ROI (1-2). Final wholefanning PyT get through the 3 ROIs manually positioned in 410 participants (3-4).

\section{Figure 3}

Overview of the whole-fanning PyT atlas (L: left; R: right). On the left side, concatenation of the 410 CSTs warped in the MNI space. Redundant streamlines have been removed using hierarchical clustering algorithm and minimal direct-flip distance operator set at $2 \mathrm{~mm}$. On the right side, tridimensional envelop of the probabilistic PyT atlas in the MNI space thresholded at $10 \%$ (details in Figure 5 and 6). Displays made with Surf Ice (left, www.nitrc.org/projects/surfice/) and MRIcroGL (right, www.nitrc.org/projects/mricrogl/). 


\section{Figure 4}

A. Illustration of the distribution of the concatenated PyT streamlines of the 410 participants colored by their gyral termination. B. Proportion of the concatenated frontal and parietal PyT streamlines. Bundle displays made with MI-BRAIN (www.imeka.ca/mi-brain). A: anterior; P: posterior.

\section{Figure 5}

Probabilistic map of the PyT atlas in different coronal (top) and axial (bottom) section in MNI space. The color bar indicates the frequency of voxels containing the PyT from 0 to $100 \%$ of the 410 participants. For example, a value of 50 in a voxel means that 205 out of 410 individual PyTs are passing through this voxel. L: left; R: right.

\section{Figure 6}

Tri-dimensional frontal view of the probabilistic PyT volumes at different thresholds from $10 \%$ to $90 \%$. The color bar indicates the frequency of voxels containing the PyT from 0 to $100 \%$ of the 410 participants. For example, 50\% means that 205 out of 410 individual PyTs are included in the volume. L: left; $R$ : right. Displays made with MRIcroGL (www.nitrc.org/projects/mricrogl/).

\section{Supplementary Figures}

Coronal, axial and sagittal plates of the concatenated PyT streamlines of the 410 participants (see Figure 3) colored by their frontal and parietal cortical termination. 


\section{References}

Angstmann S, Madsen KS, Skimminge A, Jernigan TL, Baare WF, Siebner HR (2016) Microstructural asymmetry of the corticospinal tracts predicts right-left differences in circle drawing skill in right-handed adolescents. Brain Struct Funct 221:4475-4489.

Archer DB, Vaillancourt DE, Coombes SA (2017) A Template and Probabilistic Atlas of the Human Sensorimotor Tracts using Diffusion MRI. Cereb Cortex:1-15.

Armand J (1982) The Origin, Course and Terminations of Corticospinal Fibers in Various Mammals. In: Progress in Brain Research (Kuypers HGJM, Martin GF, eds), pp 329360: Elsevier.

Avants BB, Tustison NJ, Wu J, Cook PA, Gee JC (2011) An open source multivariate framework for $n$-tissue segmentation with evaluation on public data. Neuroinformatics 9:381-400.

Bigourdan A, Munsch F, Coupe P, Guttmann CR, Sagnier S, Renou P, Debruxelles S, Poli M, Dousset V, Sibon I, Tourdias T (2016) Early Fiber Number Ratio Is a Surrogate of Corticospinal Tract Integrity and Predicts Motor Recovery After Stroke. Stroke 47:1053-1059.

Bürgel U, Amunts K, Hoemke L, Mohlberg H, Gilsbach JM, Zilles K (2006) White matter fiber tracts of the human brain: Three-dimensional mapping at microscopic resolution, topography and intersubject variability. Neurolmage 29:1092-1105.

Catani M, Thiebaut de Schotten M (2008) A diffusion tensor imaging tractography atlas for virtual in vivo dissections. Cortex 44:1105-1132.

Côté M-A, Garyfallidis E, Larochelle H, Descoteaux M (2015) Cleaning up the mess: tractography outlier removal using hierarchical QuickBundles clustering. In: 23rd ISMRM Annual Meeting. Toronto, Canada.

Curnes JT, Burger PC, Djang WT, Boyko OB (1988) MR imaging of compact white matter pathways. . American Journal of Neuroradiology 9:1061-1068.

Dejerine J, Dejerine-Klumpke A (1901) Anatomie des centres nerveux. Tome 2. Paris: Rueff et Cie.

Descoteaux M, Deriche R, Knosche TR, Anwander A (2009) Deterministic and probabilistic tractography based on complex fibre orientation distributions. IEEE Trans Med Imaging 28:269-286.

Dhollander T, Emsell L, Van Hecke W, Maes F, Sunaert S, Suetens P (2014) Track Orientation Density Imaging (TODI) and Track Orientation Distribution (TOD) based tractography. Neurolmage 94:312-336.

Dum RP, Strick PL (1991) The origin of corticospinal projections from the premotor areas in the frontal lobe. JNeurosci 11:667-689. 
Ebeling U, Reulen HJ (1992) Subcortical topography and proportions of the pyramidal tract. Acta Neurochir (Wien) 118:164-171.

Englander RN, Netsky MG, Adelman LS (1975) Location of human pyramidal tract in the internal capsule: anatomic evidence. Neurology 25:823-826.

Farquharson S, Tournier JD, Calamante F, Fabinyi G, Schneider-Kolsky M, Jackson GD, Connelly A (2013) White matter fiber tractography: why we need to move beyond DTI. J Neurosurg 118:1367-1377.

Galea MP, Darian-Smith I (1994) Multiple Corticospinal Neuron Populations in the Macaque Monkey Are Specified by Their Unique Cortical Origins, Spinal Terminations, and Connections. Cerebral Cortex 4:166-194.

Garyfallidis E, Brett M, Correia MM, Williams GB, Nimmo-Smith I (2012) QuickBundles, a Method for Tractography Simplification. Front Neurosci 6:175.

Garyfallidis E, Brett M, Amirbekian B, Rokem A, van der Walt S, Descoteaux M, NimmoSmith I, Dipy C (2014) Dipy, a library for the analysis of diffusion MRI data. Front Neuroinform 8:8.

Girard G, Whittingstall K, Deriche R, Descoteaux M (2014) Towars quantitative connectivity analysis: Reducing tractography biaises. Neurolmage 98:266-278.

Groisser BN, Copen WA, Singhal AB, Hirai KK, Schaechter JD (2014) Corticospinal Tract Diffusion Abnormalities Early After Stroke Predict Motor Outcome. Neurorehabilitation and Neural Repair 28:751-760.

Hau J, Sarubbo S, Houde JC, Corsini F, Girard G, Deledalle C, Crivello F, Zago L, Mellet E, Jobard G, Joliot M, Mazoyer B, Tzourio-Mazoyer N, Descoteaux M, Petit L (2017) Revisiting the human uncinate fasciculus, its subcomponents and asymmetries with stem-based tractography and microdissection validation. Brain Struct Funct 222:1645-1662.

Hervé P-Y, Cox EF, Lotfipour AK, Mougin OE, Bowtell RW, Gowland PA, Paus T (2011) Structural properties of the corticospinal tract in the human brain: a magnetic resonance imaging study at 7 Tesla. Brain Struct Funct 216:255-262.

Hervé P-Y, Leonard G, Perron M, Pike B, Pitiot A, Richer L, Veillette S, Pausova Z, Paus T (2009) Handedness, motor skills and maturation of the corticospinal tract in the adolescent brain. Human Brain Mapping 30:3151-3162.

Jane JA, Yashon D, DeMyer W, Bucy PC (1967) The contribution of the precentral gyrus to the pyramidal tract of man. J Neurosurg 26:244-248.

Jbabdi S, Behrens TEJ (2012) Specialization: the connections have it. Nat Neurosci 15:171172.

Jbabdi S, Sotiropoulos SN, Haber SN, Van Essen DC, Behrens TE (2015) Measuring macroscopic brain connections in vivo. Nat Neurosci 18:1546-1555. 
Jones DK, Knosche TR, Turner R (2013) White matter integrity, fiber count, and other fallacies: the do's and don'ts of diffusion MRI. Neuroimage 73:239-254.

Kretschmann HJ (1988) Localisation of the corticospinal fibres in the internal capsule in man. J Anat 160:219-225.

Kumar A, Juhasz C, Asano E, Sundaram SK, Makki MI, Chugani DC, Chugani HT (2009) Diffusion Tensor Imaging Study of the Cortical Origin and Course of the Corticospinal Tract in Healthy Children. American Journal of Neuroradiology 30:1963-1970.

Kwon HG, Hong JH, Jang SH (2011) Anatomic location and somatotopic arrangement of the corticospinal tract at the cerebral peduncle in the human brain. Am $\mathrm{J}$ Neuroradiol 32:2116-2119.

Maier-Hein $\mathrm{KH}$ et al. (2017) The challenge of mapping the human connectome based on diffusion tractography. Nature Communications 8:1349.

Mayka MA, Corcos DM, Leurgans SE, Vaillancourt DE (2006) Three-dimensional locations and boundaries of motor and premotor cortices as defined by functional brain imaging: A meta-analysis. Neurolmage 31:1453.

Mazoyer B, Mellet E, Perchey G, Zago L, Crivello F, Jobard G, Delcroix N, Vigneau M, Leroux G, Petit L, Joliot M, Tzourio-Mazoyer N (2016) BIL\&GIN: A neuroimaging, cognitive, behavioral, and genetic database for the study of human brain lateralization. Neuroimage 124 Part B:1225-1231.

Mirowitz S, Sartor K, Gado M, Torack R (1989) Focal signal-intensity variations in the posterior internal capsule: normal MR findings and distinction from pathologic findings. Radiology 172:535-539.

Nathan PW, Smith MC (1955) Long descending tracts in man: I. review of present knowledge. Brain 78:248-303.

Nieuwenhuys R, Voogd J, van Huijzen C (2008) The human central nervous system, 4th Edition. Berlin: Springer-Verlag.

Nyberg-Hansen R, Rinvik E (1963) Some comments on the pyramidal tract, with special reference to its individual variations in man. Acta Neurologica Scandinavica 39:1-30.

Penfield W, Boldrey E (1937) Somatic motor and sensory representation in the cerebral cortex of man as studied by electrical stimulation. Brain 60:389-443.

Rheault F, St-Onge E, Chenot Q, Sidhu J, Petit L, Descoteaux M (2017) Bundle-specific tractopgraphy. In: Computational Diffusion MRI, MICCAI Workshop. Québec, Canada: Springer, CDMRI'17.

Rojkova K, Volle E, Urbanski M, Humbert F, Dell'Acqua F, Thiebaut de Schotten M (2016) Atlasing the frontal lobe connections and their variability due to age and education: a spherical deconvolution tractography study. Brain Struct Funct 221:1751-1766. 
Ross ED (1980) Localization of the pyramidal tract in the internal capsule by whole brain dissection. Neurology 30:59-64.

Seo JP, Jang SH (2013) Different Characteristics of the Corticospinal Tract According to the Cerebral Origin: DTI Study. AJNR.

Smith SM, Jenkinson M, Woolrich MW, Beckmann CF, Behrens TEJ, Johansen-Berg $H$, Bannister PR, De Luca M, Drobnjak I, Flitney DE, Niazy RK, Saunders J, Vickers J, Zhang Y, De Stefano N, Brady JM, Matthews PM (2004) Advances in functional and structural MR image analysis and implementation as FSL. Neurolmage 23, Supplement 1:S208-S219.

Thiebaut de Schotten M, Ffytche D, Bizzi A, Dell'acqua F, Allin M, Walshe M, Murray R, Williams S, Murphy DGM, Catani M (2011) Atlasing location, asymmetry and intersubject variability of white matter tracts in the human brain with MR diffusion tractography. Neurolmage 54:49-59.

Yagishita A, Nakano I, Oda M, Hirano A (1994) Location of the corticospinal tract in the internal capsule at MR imaging. Radiology 191:455-460.

Yendiki A, Panneck P, Srinivasan P, Stevens A, Zollei L, Augustinack J, Wang R, Salat D, Ehrlich S, Behrens T, Jbabdi S, Gollub R, Fischl B (2011) Automated probabilistic reconstruction of white-matter pathways in health and disease using an atlas of the underlying anatomy. Front Neuroinform 5:23.

Zhang Y, Zhang J, Oishi K, Faria AV, Jiang H, Li X, Akhter K, Rosa-Neto P, Pike GB, Evans A, Toga AW, Woods R, Mazziotta JC, Miller MI, van Zijl PCM, Mori S (2010) Atlasguided tract reconstruction for automated and comprehensive examination of the white matter anatomy. Neurolmage 52:1289-1301.

Zolal A, Vachata P, Hejčl A, Bartoš R, Malucelli A, Nováková M, Derner M, Sameš M (2012) Anatomy of the supraventricular portion of the pyramidal tract. Acta Neurochir (Wien) 154:1097-1104. 
Figure 1

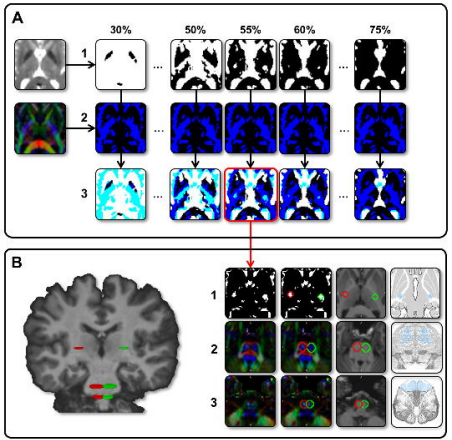


Figure 2
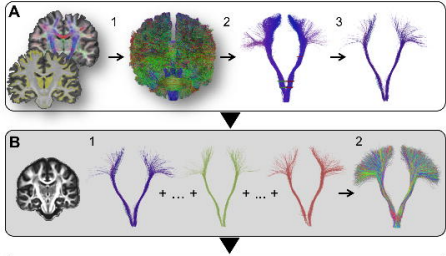

c
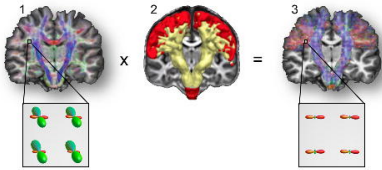

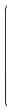

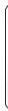

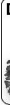
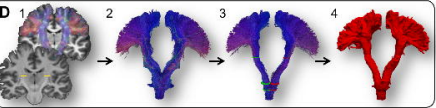
Figure 3

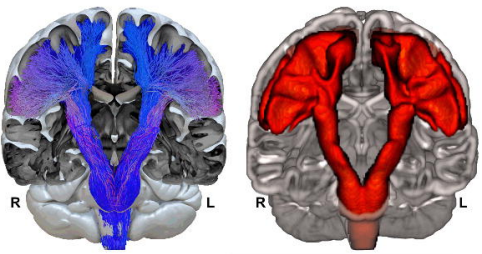




\section{Figure 4}

A. Lateral view
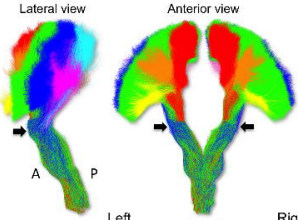

Left

Right

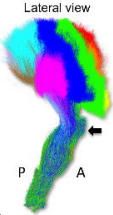

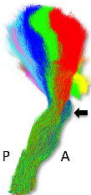

Medial view

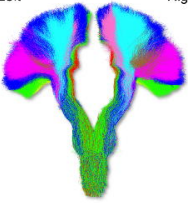

Posterior view

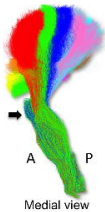

B. Proportion of PyT streamlines per gyrus (in \%) left right Left Right
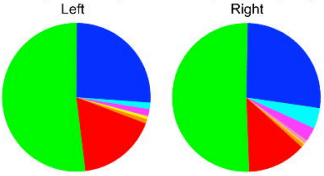

$\square \mathrm{PrC} \quad 52.0 \quad 50.9$

$\square$ PoC $26.0 \quad 27.0$

$\square$ SFG $\quad 17.4 \quad 12.8$

$\square$ MFG $\quad 0.9 \quad 0.7$

$\square$ IFG $\quad 0.6 \quad 0.2$

$\square$ SMG $\quad 1.5 \quad 2.9$

$\square$ SPG $\quad 1.4 \quad 4.5$

$\begin{array}{lll}\square \text { AG } & 0.0 \quad 0.1\end{array}$

$\begin{array}{lll}\square \mathrm{PCu} & 0.1 & 0.8\end{array}$ 


\section{Figure 5}
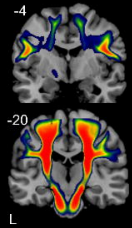

$-24$

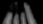

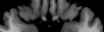
Exars

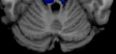

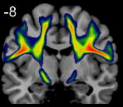

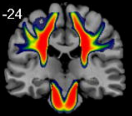

(3) and

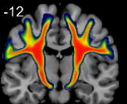

Fand

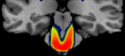

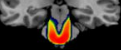

J.

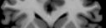
E Solo

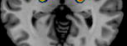
है

48

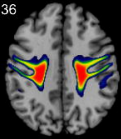

-16 of

(2)

-32 and

Es

Cand

$\sin \mathrm{R}$

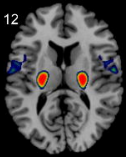

60

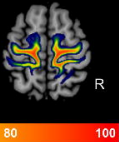


Figure 6
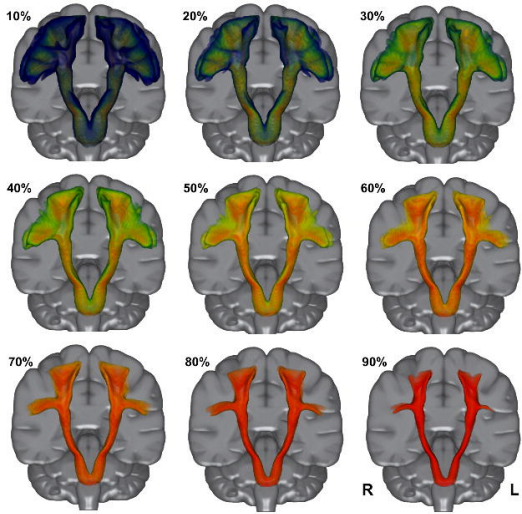

0 